\title{
Effective properties of pore network elements derived from reactive transport in individual pores
}

\author{
Qingrong Xiong, Andrey P Jivkov \\ Research Centre for Radwaste \& Decommissioning and Modelling \& Simulation Centre, Dalton \\ Nuclear Institute, The University of Manchester, UK, M13 9PL
}

qingrong.xiong@manchester.ac.uk; andrey.Jivkov@manchester.ac.uk

Keywords: Reactive transport, pore scale, coarse graining, pore network

\begin{abstract}
Adsorption of solutes in porous media is typically represented as an equilibrium process. The adsorption process is determined by the concentration of the solute in the fluid next to the solid grain in individual pores. The concentration near the solid surface is different from the average concentration within the pore due to the development of concentration gradients within the pore space. Simulations with pore network models are usually based on the average concentration in individual pore throats. To advance the realism of such models, we develop a relationship between pore-scale adsorption coefficients and corresponding coarse-grained adsorption parameters. A numerical scheme is proposed: firstly the solute transport within a single pore is simulated undergoing equilibrium adsorption at the pore wall, and secondly flux-averaged concentration breakthrough curves are obtained. By fitting the breakthrough curves, the coarse-grained adsorption parameters are determined from the pore-scale hydraulic and adsorption parameters.
\end{abstract}

\section{Introduction}

Pore network models are one of the appealing approaches to study transport properties in porous materials [1-3]. Pore network models usually consist of a set of pores and a set of throats connecting neighboring pores. Previous works have demonstrated clearly the importance of the geometric properties of the porous media, such as the locations of pores and throats, the distributions of sizes and shapes of pores and throats [4-6]. Most of early works on pore-scale modeling assumed that the throats were cylinders with a circular cross-section or considered to be zero-volume connections between pore chambers. Pores were either not modeled explicitly (they simply represent throat junctions), or were spherical, cube or cylindrical in shape. The physicochemical processes in pore network are usually described by simple relationships within the pore network model. For example, diffusion is represented by Fick's law, adsorption is assumed to follow an equilibrium relationship and equilibrium dissolution is explained by Raoult's law [2,3]. These larger scale descriptions of transport, however, do not consider some important aspects of smaller scale behavior which may lead to discrepancies between observation and theory [7-9]. Hence, it is important to include subscale mass transport effects in the description of porous systems which is essential to develop theoretically sound equations for describing modeling mass transport at the larger scales.

Reaction processes such as adsorption and mineral dissolution have a great influence on the transport behavior in porous materials $[10,11]$. These processes typically occur at fluid-fluid interfaces or solid-fluid boundaries. The adsorption is represented as an equilibrium process where the chemical potential of the solute in the fluid next to the solid grain is equal to the chemical potential of the solute adsorbed to the grain [12]. This assumption is probably acceptable in most interactions at the pore boundaries. However, this is not the case in pore network models where the simulation is based on the average solute concentrations in each pore. As the solute concentration close to the wall is different 
from the average concentration in the bulk fluid, the adsorbed solute concentration closed to the adsorbed site may not be in equilibrium with the average concentration of solution [13, 14]. The kinetic relationship is usually applied between average concentration of dissolved and adsorbed solutes in porous medium especially for those with microscopically heterogeneous structure.

This aim of this work is to develop up-scaled (coarse-grained) relationships for transport properties in pore network elements from reactive transport in individual pores. The relations for coarse-grained transport coefficients are expected to be valid for a solute that, at the pore scale, undergoes diffusion and advection as well as first-order equilibrium action at the solid surface. The steady state flow and transient adsorption in a single pore at the pore scale are simulated numerically. The resulting breakthrough curves from this single pore are compared to the solution of the 1-D continuum scale transport equation to estimate the coarse-grained adsorption parameters.

\section{Theory}

This work is focused on the reaction which takes place at the interface between the fluid and solid phases within a porous medium. The mass conservation in each pore is governed by the local equation

$$
\nabla \mathrm{v}=0 \text { in } \Omega_{\mathrm{F}}
$$

where the porous medium $\Omega$ is divided into a solid volume $\Omega_{\mathrm{s}}$ and a fluid volume $\Omega_{\mathrm{F}}$. $\Gamma$ is defined as the interface between these two volumes, $v$ is the local velocity of the fluid.

Assuming that the flow is steady and that inertial effects can be neglected, the fluid phase motion is governed by the Stokes equation

$$
\mu \nabla^{2} \mathrm{v}=\nabla \mathrm{p} \text { in } \Omega_{\mathrm{F}}
$$

where $\mu$ and $p$ are the dynamic viscosity and the local pressure of the fluid, respectively.

At the rock-fluid interface, the no slip boundary condition applies

$$
\mathrm{v}=0 \text { on } \Gamma
$$

The solute concentration $c$ is governed by the classical convection-diffusion equation

$$
\frac{\partial \mathrm{c}}{\partial \mathrm{t}}+\nabla(\mathrm{cv}-\mathrm{D} \nabla \mathrm{c})=0 \text { in } \Omega_{\mathrm{F}}
$$

At a point on the pore boundary, adsorption causes a flux of solute from the fluid to the solid phase. This gives rise to an increase in the mass density of adsorbed solutes. The rate of adsorption is equal to the solute mass flux normal to the pore boundary

$$
(\mathrm{cv}-\mathrm{D} \nabla \mathrm{c}) \cdot n=\frac{\partial s}{\partial t}
$$

where $n$ is the unit vector normal to $\Gamma$ pointing into the solid phase, $s$ is the mass of adsorbed solute per unit area of the solid grains. 
Thus the coupled problem of flow (1)-(3), transport (4) and reaction (5) needs to be solved on the pore scale.

To analyze the scale dependence of adsorption process, we have developed two models: a) a single pore model in order to simulate details of transport within a pore; $b$ ) an equivalent upscaled 1D model for the cross sectional averaged concentration applicable to pore networks. These models allow us to investigate some of the assumptions made in our coarse-graining approach from events in a single pore to a pore in a network, as well as to verify results of that approach. The program of flexpde (http://www.pdesolutions.com/) has been used to numerically solve this set of equations.

\section{Formulation of a single pore model}

Consider a long single pore with a constant circular cross section with radius $R_{0}$. We assume fully developed, steady-state, laminar flow in the pore (Poiseuille flow) so that the velocity distribution is given by (in Fig.1):

$$
v(r)=2 \bar{v}\left[1-\left(\frac{r}{R_{0}}\right)^{2}\right]
$$

where $v(r)$ is the local fluid velocity, $\bar{v}$ is the average flow velocity, $r$ is the radial coordinate, and $R_{0}$ is the radius of the cylinder. In the single pore model, adsorption occurs only at the wall of pore. In this work, we do not take into the account changes of pore radius induced by the adsorption process. The mass transport in the pore is then given by

$$
\frac{\partial c}{\partial t}+2 \bar{v}\left(1-\left(\frac{r}{R_{0}}\right)^{2}\right) \frac{\partial c}{\partial z}=D_{0}\left[\frac{\partial^{2} c}{\partial z^{2}}+\frac{1}{r} \frac{\partial}{\partial r}\left(r \frac{\partial c}{\partial r}\right)\right]
$$

where $\mathrm{z}$ is the axial direction along the pore. In the pore, the wall acts as an adsorbent and therefore, the adsorption relation must appear in the boundary condition to the differential Equation (7). In literature, the solid - fluid interface for adsorptive solute is often assumed to be a diffusion-limited boundary, described by $r=D \partial c / \partial n$, where $n$ is the unit vector normal to the boundary. Since the solid - fluid interface is assumed to be a non-slip boundary where the fluid velocity is zero, from definition of the solute flux, we know that $D \partial c / \partial n$ is actually the solute flux pointing to the interface:

$$
\frac{\partial s}{\partial t}=-D_{0} \frac{\partial c}{\partial r}
$$

where $s$ is adsorbed mass per unit area, $D_{0}$ is the molecular diffusion coefficient for a specific species which can by calculated by the Einstein-Stokes equation [2]. The linear equilibrium adsorption is assumed to occur at the wall which can be characterized by the distribution coefficient, $k_{d}$ :

$$
s=\left.k_{d} c\right|_{s}
$$

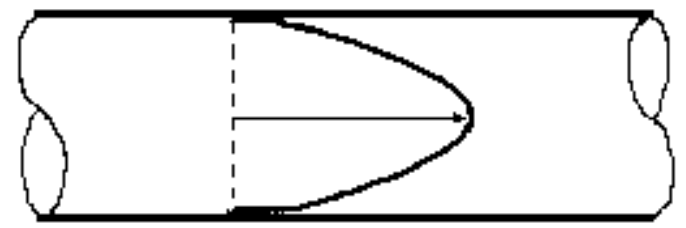


Fig.1 Conceptual representation for the single pore model. The velocity profile is assumed to be parabolic.

Then the following dimensionless parameters and variables are introduced:

$$
c^{*}=\frac{c}{c_{0}} ; r^{*}=\frac{r}{R_{0}} ; z^{*}=\frac{z}{R_{0}} ; t^{*}=\frac{t D_{0}}{R_{0}^{2}} ; P e_{p}=\frac{\bar{v} R_{0}}{D_{0}} ; s^{*}=\frac{s}{c_{0} R_{0}} ; k=\frac{k_{d}}{R_{0}}
$$

where $c_{0}$ is concentration at the inlet boundary, $P e_{p}$ is defined as the pore-scale Peclet number, which expresses the ratio between the magnitude of the advective and diffusive transport terms. Substituting in Equation (7) gives the pore-scale dimensionless mass transport equation

$$
\frac{\partial c^{*}}{\partial t^{*}}+2 P e_{p}\left(1-r^{* 2}\right) \frac{\partial c^{*}}{\partial z^{*}}=\frac{\partial^{2} c^{*}}{\partial z^{* 2}}+\frac{1}{r^{*}} \frac{\partial}{\partial r^{*}}\left(r^{*} \frac{\partial c^{*}}{\partial r^{*}}\right)
$$

The dimensionless boundary conditions are

$$
\begin{aligned}
& \text { at } z^{*}=0, c^{*}=1.0 \\
& \text { at } z^{*}=\infty, \frac{\partial c^{*}}{\partial z^{*}}=0 \\
& \text { at } r^{*}=0, \frac{\partial c^{*}}{\partial r^{*}}=0 \\
& \text { at } r^{*}=1, \frac{\partial c^{*}}{\partial r^{*}}=-\frac{\partial s^{*}}{\partial t^{*}} \\
& \text { at } r^{*}=1, \frac{\partial c^{*}}{\partial r^{*}}=-k \frac{\partial c^{*}}{\partial t^{*}}
\end{aligned}
$$

The solution of Equations (11)-(15) results in a concentration field $\mathrm{c}^{*}\left(\mathrm{z}^{*} ; \mathrm{r}^{*} ; \mathrm{t}^{*}\right)$ for different values of Peclet number $\left(P e_{p}\right)$ and dimensionless distribution coefficient $(k)$.

\section{Formulation of an upscaled 1-D model}

The 1D average concentration field, $\mathrm{c}^{*}\left(\mathrm{z}^{*} ; \mathrm{t}^{*}\right)$, is obtained from averaging the pore-scale concentration over the cross section of the pore. The reaction between solute and solid is assumed to be first-order kinetic, taking place at the fluid - solid interface. We use accumulation rate $r$ to represent the net amount of solute adsorbed to an interface between solid and fluid over $\delta t$ period. Referring to Fig. 1, if we denote the adsorption rate on the interface between the fluid and solid by $k_{a}$ and the desorption rate by $k_{b}, r$ can then be expressed as $r=k_{a} c-k_{b} s$, where $c$ is the concentration of the dissolved solute in the fluid cell and $s$ is the amount of solute adsorbed to the interface. The governing equations for the 1D transport of a kinetically adsorbing solute are

$$
\frac{\partial c^{*}}{\partial t^{*}}+P e \frac{\partial c^{*}}{\partial z^{*}}=\frac{\partial^{2} c^{*}}{\partial z^{* 2}}-\frac{\partial \overline{s^{*}}}{\partial t^{*}}
$$




$$
\begin{aligned}
& \frac{\partial s^{*}}{\partial t^{*}}=k_{a}^{*} c^{*}-k_{b}^{*} s^{*} \\
& k_{a}^{*}=\frac{k_{a} R_{0}^{2}}{D_{0}} \\
& k_{b}^{*}=\frac{k_{b} R_{0}^{2}}{D_{0}}
\end{aligned}
$$

where $P e=v R_{0} / D_{L}$, which involves the average flow velocity $v$ and the longitudinal dispersion coefficient, $D_{L}$. Note that $P e$ is different from the pore-scale $P e_{p}$, which is based on a diffusion coefficient. Further, $k_{a}{ }^{*}$ is dimensionless adsorption rate coefficients and $k_{b}{ }^{*}$ is the dimensionless desorption rate coefficients, $k_{a}$ and $k_{b}$ are the corresponding dimensional adsorption rate coefficients and desorption rate coefficients, respecitvely.

\section{Results and discussions}

Equations (17) - (20) contain three parameters: $P e, k_{a}^{*}, k_{b} *$. We have evaluated these parameters by fitting the solution of this set of equations to the average breakthrough concentration, $\mathrm{c}^{*}\left(\mathrm{z}^{*} ; \mathrm{t}^{*}\right)$, obtained from the single pore model for various values of pore-scale Peclet number (Pep) and $k$ (Fig.2 and Fig.3). The relationships between the three upscaled parameters $\left(P e, k_{a}^{*}, k_{b}{ }^{*}\right)$ and their corresponding pore-scale parameters $\left(P e_{p}\right.$ and $\left.k\right)$ have been obtained. We have used the cross-sectional averaged concentrations from the single pore model to find corresponding coarse-grained adsorption parameters.

Here we assume that the dispersion is not influenced by the adsorption process. Therefore, the coarse-grained Peclet number $(P e)$ can be obtained by the case of a non-adsorbing solute (i.e., $k=0)$ in Fig.2. This is achieved by fitting the breakthrough curve from the single pore model to the solution of the $1 \mathrm{D}$ transport equation (Equation (11)) with no adsorption ((i.e., $\left.k_{a}{ }^{*}=k_{b}{ }^{*}=0\right)$.

$$
P e=P e_{p}
$$

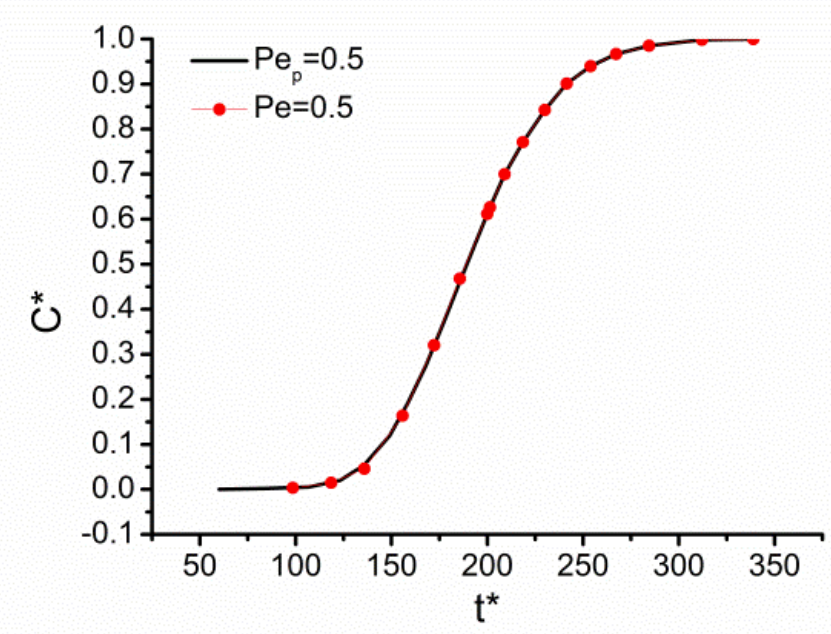

Fig.2 Breakthrough curve of average concentration from local single pore model (red line) and 1-D tube model (black line) with no adsorption. 
The excellent agreement in Fig. 2 indicates the accuracy of the numerical codes in capturing the tranport behavior within the pore and verifying the assumption for this problem as well.

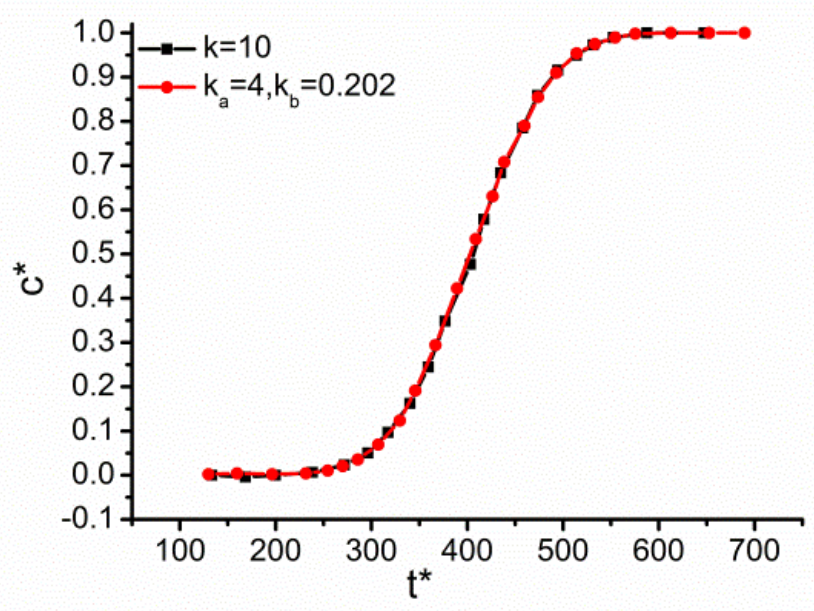

Fig.3 Breakthrough curve of average concentration from local single pore model (red line) and 1-D tube model (black line) when $\mathrm{Pe}=\mathrm{Pep}=5$.

As the upscaled Peclet number has been estimated, only two upscaled parameters, $k_{a}{ }^{*}$ and $k_{b}{ }^{*}$, are left to be determined as a function of pore-scale parameters. The flexpde program was used to solve Equations (17) and (18) and fit to the breakthrough curves. Fig.4 and Fig.5 show an example fit to the breakthrough curves and the corresponding parameters. Through this fitting process, the relationship between $k_{a}{ }^{*}, k_{b}{ }^{*}$ and $P e_{p}, k$ is summarized as follows:

$$
\begin{aligned}
& k_{a}^{*}=4\left(0.92-e^{-0.95 k}\right) P e_{p}^{0.07} \\
& k_{b}^{*}=\frac{9 P e_{p}^{0.05}}{1.5+4.5 k}-e^{-1.1 k}
\end{aligned}
$$

Equations (22) and (23) can be converted to a function of dimensional parameters via Equation (10):

$$
\begin{aligned}
& k_{a}=4\left(0.92-e^{-0.95 \frac{k_{\mathrm{d}}}{R_{0}}}\right)(\bar{v})^{0.07} \frac{D_{0}^{0.93}}{R_{0}^{1.93}} \\
& k_{b}=\left[\frac{9\left(\frac{\bar{v} R_{0}}{D_{0}}\right)^{0.05}}{1.5+4.5 \frac{k_{\mathrm{d}}}{R_{0}}}-e^{-1.1 \frac{k_{\mathrm{d}}}{R_{0}}}\right]\left(\frac{D_{0}}{R_{0}^{2}}\right)
\end{aligned}
$$

From Equations (24) and (25), it can be observed that $k_{a}{ }^{*}$ and $k_{b}{ }^{*}$ are strongly dependent on the molecular diffusion coefficient, the geometry of the pore space and the pore scale distribution coefficient, but weakly dependent on the avreage velocity. The relations for upscaled transport coefficients are suitable for use in pore network models where pore scale relationships are required to model adsorbing solute transport in individual pore throats. 


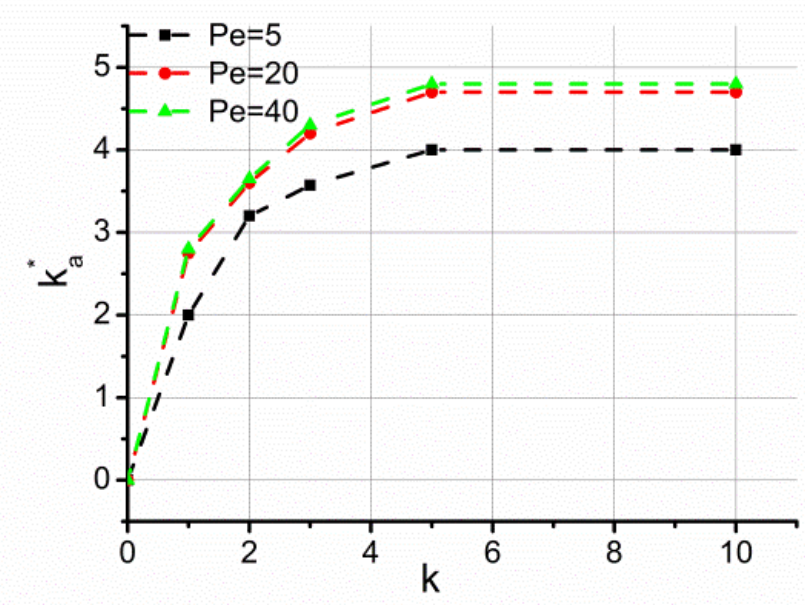

Fig.4 The relationship between upscaled $\mathrm{k}_{\mathrm{a}}$ and $\mathrm{k}$.

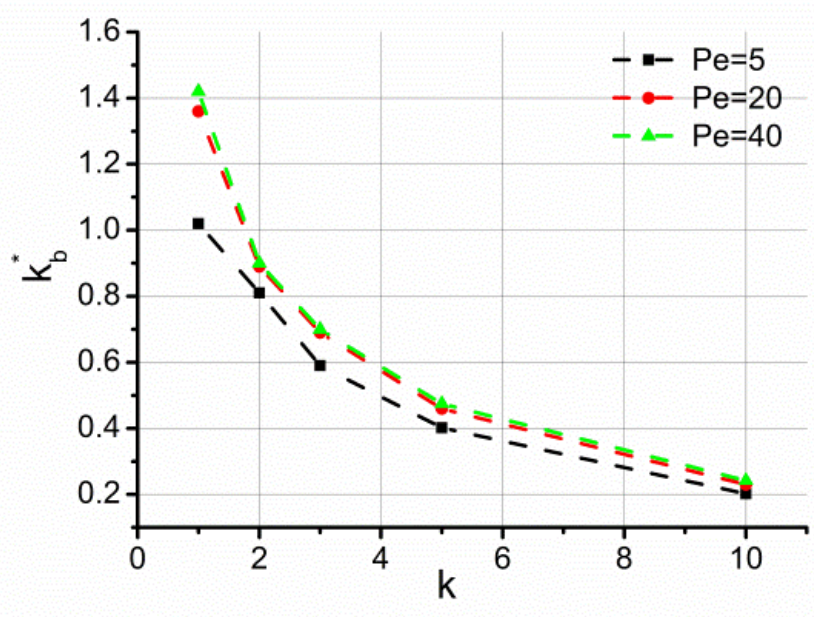

Fig.5 The relationship between upscaled $\mathrm{k}_{\mathrm{b}}{ }^{*}$ and $\mathrm{k}$.

\section{Conclusions}

This work shows that it is necessary to employ a kinetic description at the larger scale even if there is equilibrium adsorption at the pore wall (or at the grain surface). This result was obtained through numerical averaging from pore to the 1D scale. The larger scale kinetic adsorption parameters are obtained as a function of pore scale parameters such as Peclet number and distribution coefficient. The coarse-grained adsorption parameters are found to depend weakly on the fluid velocity, but to depend strongly on the pore radii, diffusion coefficient and the the pore-scale distribution coefficient.

The coarse-grained transport coefficients in Equations (21), (24) and (25), can be applied to pore network models where modeling reactive solute transport in individual pore throats is required. Using pore-network models allows for scaling up from the pore scale to the scale of a statistically representative volume element and even the core scale with sufficient computational power.

\section{References}

[1] Blunt MJ. Flow in porous media - pore-network models and multiphase flow. Current opinion in colloid \& interface science. 2001;6:197-207. 
[2] Xiong Q, Jivkov AP, Yates JR. Discrete modelling of contaminant diffusion in porous media with sorption. Microporous and Mesoporous Materials. 2014;185:51-60.

[3] Jivkov AP, Xiong Q. A Network Model for Diffusion in Media with Partially Resolvable Pore Space Characteristics. Transport in Porous Media. 2014;105:83-104.

[4] Oren P. Pore-scale network modelling of waterflood residual oil recovery by immiscible gas flooding. SPE/DOE Improved Oil Recovery Symposium1994.

[5] Blunt M, King MJ, Scher H. Simulation and theory of two-phase flow in porous media. Physical Review A. 1992;46:7680.

[6] Knackstedt MA, Sheppard AP, Pinczewski W. Simulation of mercury porosimetry on correlated grids: Evidence for extended correlated heterogeneity at the pore scale in rocks. Physical review E. 1998;58:R6923.

[7] Bryant SL, Thompson KE. Theory, modeling and experiment in reactive transport in porous media. Current opinion in colloid \& interface science. 2001;6:217-22.

[8] Raje DS, Kapoor V. Experimental study of bimolecular reaction kinetics in porous media. Environmental science \& technology. 2000;34:1234-9.

[9] Gramling CM, Harvey CF, Meigs LC. Reactive transport in porous media: A comparison of model prediction with laboratory visualization. Environmental science \& technology. 2002;36:2508-14.

[10] Serrano SE. Propagation of nonlinear reactive contaminants in porous media. Water resources research. 2003;39.

[11] Acharya R, Van der Zee S, Leijnse A. Transport modeling of nonlinearly adsorbing solutes in physically heterogeneous pore networks. Water resources research. 2005;41.

[12] Weber WJ, McGinley PM, Katz LE. Sorption phenomena in subsurface systems: concepts, models and effects on contaminant fate and transport. Water Research. 1991;25:499-528.

[13] Binning PJ, Celia M. Pseudokinetics arising from the upscaling of geochemical equilibrium. Water resources research. 2008;44.

[14] Li L, Steefel CI, Yang L. Scale dependence of mineral dissolution rates within single pores and fractures. Geochimica et Cosmochimica Acta. 2008;72:360-77. 